\title{
A NEW SURVEY OF GLOBULAR CLUSTER STRUCTURAL AND LUMINOSITY PARAMETERS
}

\author{
B. Cameron Reed \\ St. Mary's University, Halifax \\ Charles J. Peterson \\ University of Missouri
}

We have made an analysis of the visual photometric data contained in the Catalogue of Concentric Aperture UBVRI Photoelectric Photometry of Globular Clusters (Peterson 1986). Structural parameters have been obtained by use of the Simplex algorithm of Caceci and Cacheris (1984) to fit the model curves of King (1966) to the run of cluster luminosity with radius. We find that concentric aperture photometry alone can be used to determine globular cluster core radii and central surface brigtnesses reliably. Application of this techique, however, is limited to about two-thirds of the known clusters of the Galaxy because no or inadequate numbers of photometric measurements exist for the remaining clusters. Accurate determination of cluster concentration classes still requires use of other types of data, such as star counts.

Comparison of our derived cluster structural and luminosity parameters with those determined in other studies gives the following conclusions :

a) The core radii given by Peterson and King (1975) and Peterson (1976) appear to be systematically too large by approximately 0.1 dex in $\log \left(r^{\prime}\right)$.

b) Our core radii are in good agreement with the values that Kron, Hewitt, and Wassermann (1983) obtained from surface photometry of electronic camera exposures. The estimated r.m.s. scatter about an equality relationship is $0.08 \mathrm{dex}$, implying an intrinsic error of 0.06 dex in our results if equal weight is given to the data of the two studies.

c) From comparison to our core radii, the smallest core radii obtained by Webbink (1984) appear to be underestimated in size; his larger core

J. E. Grindlay and A. G. Davis Philip (eds.),

The Harlow-Shapley Symposium on Globular Cluster Systems in Galaxies, 489-490.

(C) 1988 by the IAU- 
radii appear to be slightly overestimated in size. Of the 69 clusters studied by Kron, Hewitt, and Wassermann, their values of $\log \left(r^{\prime}{ }_{c}\right)$ for 53 clusters are identical with the values tabulated by Webbink; thus, a systematic problem appears to exist only in those values of core radii obtained by Webbink from consideration of other data (either the concentric aperture photometry or star counts).

d) Our analysis (as well as that of Kron, Hewitt, and Wassermann) does not obtain values of cluster concentration class $c=\log \left(r_{t} / r_{c}\right)$ that are of high statistical accuracy, although our current results appear to be in best agreement with the studies of Peterson and King (1975) and Peterson (1976).

e) Our results for central surface brightness appear we1l-behaved and agree well with the prior values obtained by Peterson and King, Peterson, and by Webbink over a range of 5 to 14 visual magnitudes per square minute of arc. The scatter about equality is significantly smaller for the comparison with Webbink.

f) Although concentration class appears to be poorly defined in our calculations, total magnitudes which are strongly dependent upon the adopted value of $c$ are reasonably well-behaved. The majority of our values agree with those in the Peterson and King (1975) and Peterson (1976) studies and with those of Webbink, though the r.m.s. scatter about equality appears larger in the comparison with Webbink. In a number of cases in which the concentric aperture data span an insufficient radial range to define c precisely, our method appears to overestimate cluster concentration and hence overestimates cluster brightness.

One of us (C.J.P.) wishes to acknowledge the support and hospitality of the Berkeley Astronomy Department and the Dominion Astrophysical Observatory where this work was performed during a sabbatical leave from the University of Missouri. B.C.R. is supported in part by a grant from the Natural Sciences and Engineering Research Council of Canada.

\section{REFERENCES}

Caceci, M. S. and Cacheris, W. P. 1984 Byte 9, \#5, 340. King, I. R. 1966 Astron. J. 71, 64.

Kron, G. E., Hewitt, A. V. and Wassermann, L. H. 1983 Publ. Astron. Soc. Pacific 96, 198 .

Peterson, C. J. and King, I. R. 1975 Astron. J. 80,427.

Peterson, C. J. 1976 Astron. J. 81, 617.

Peterson, C. J. 1986 Astron. Data Center Bull. 1, in press.

Webbink, R. F. 1984 in I.A.U. Symposium 115, Dynamics of Star Clusters, J. Goodman and P. Hut, eds., Reidel, Dordrecht, p. 541. 